\title{
Constitutive Activation of 5-Lipoxygenase in the Lungs of Patients with Idiopathic Pulmonary Fibrosis
}

\author{
Jerome Wilborn, Marc Bailie, Michael Coffey, Marie Burdick, Robert Strieter, and Marc Peters-Golden \\ Division of Pulmonary and Critical Care Medicine, University of Michigan Medical Center, Ann Arbor, Michigan 48109
}

\begin{abstract}
Idiopathic pulmonary fibrosis (IPF) is a progressive disorder characterized by inflammation, fibroblast proliferation, and accumulation of extracellular matrix proteins. Leukotrienes (LTs) are pro-inflammatory and pro-fibrogenic mediators derived from the 5-lipoxygenase (5-LO) pathway of arachidonic acid metabolism. They are thought to play a role in a number of disease processes, but have received relatively little attention in investigations into the pathogenesis of IPF. In this study, we measured the levels of immunoreactive LTs $B_{4}$ and $C_{4}$ in homogenates of lung tissue obtained from patients with newly diagnosed, untreated IPF, as compared to levels measured in homogenates of uninvolved nonfibrotic lung tissue from patients undergoing resectional surgery for bronchogenic carcinoma. Compared to homogenates of nonfibrotic control lung, homogenates from IPF patients contained 15 -fold more $\mathrm{LTB}_{4}$ and 5-fold more $\mathrm{LTC}_{4}$. IPF homogenate levels of $\mathrm{LTB}_{4}$ were significantly correlated with histologic indices of both inflammation $(r=0.861)$ and fibrosis $(r=0.926)$. Activation of 5-LO is known from in vitro studies to be associated with localization of the enzyme at the nuclear membrane. Immunohistochemical staining for 5-LO protein in alveolar macrophages (AMs) demonstrated that such an "activated" localization pattern was significantly more frequent in IPF lung (19.2 \pm $3.3 \%$ of cells) than in control lung $(9.3 \pm 0.9 \%)$; this localization pattern was rarely seen (3.2\%) in sections from a truly normal transplant donor lung. Consistent with these data, AMs obtained from IPF patients by bronchoalveolar lavage, purified by adherence, and cultured in the absence of a stimulus for $16 \mathrm{~h}$ elaborated significantly greater amounts of $\mathrm{LTB}_{4}$ and $\mathrm{LTC}_{4}$ than did control AMs obtained from normal volunteers. These data indicate that the 5-LO pathway is constitutively activated in the lungs of patients with IPF, and the AM represents at least one cellular source of LT overproduction in this disorder. We speculate that LTs participate in the pathogenesis of IPF, and their overproduction in this disorder may be amenable to specific pharmacotherapy. (J. Clin. Invest. 1996. 97:1827-1836.) Key words: leukotrienes $\bullet$ 5-lipoxygenase $\cdot$ alveolar macrophages $\bullet$ pulmonary fibrosis • immunohistochemistry
\end{abstract}

Address correspondence to Marc Peters-Golden, M.D., Division of Pulmonary and Critical Care Medicine, 3916 Taubman Center, University of Michigan Medical Center, Ann Arbor, MI 48109-0360. Phone: 313-936-2612; FAX: 313-764-4556; E-mail: petersm@umich.edu Received for publication 5 September 1995 and accepted in revised form 5 February 1996.

J. Clin. Invest.

(C) The American Society for Clinical Investigation, Inc.

0021-9738/96/04/1827/10 \$2.00

Volume 97, Number 8, April 1996, 1827-1836

\section{Introduction}

Idiopathic pulmonary fibrosis (IPF) $)^{1}$ is the most common of the infiltrative or interstitial disorders of the lung parenchyma. It is manifested as a restrictive ventilatory defect with impaired gas exchange, and its course is typically progressive, culminating in respiratory failure and death. Histologically, IPF is characterized by an accumulation of inflammatory cells in the alveolar space and interstitium, derangement of normal alveolar architecture, and expansion of the mesenchymal cell population with increased deposition of extracellular matrix components (1). These same clinical and histopathologic features are also observed in other interstitial lung diseases, such as those associated with asbestos exposure (2) and collagen vascular disorders (3).

Although little is known about the inciting circumstances which lead to the development of IPF, some information regarding the evolution of this lesion is available (4). An early phase of the disease likely involves an expansion of the population of resident alveolar macrophages (AMs) and the recruitment from peripheral blood of inflammatory cells such as neutrophils and eosinophils. Interactions among these recruited inflammatory cells, the resident alveolar lymphocytes and AMs, and the endothelial and epithelial cells comprising the normal alveolar wall then result in cellular injury as well as the generation of growth signals. The ultimate consequence of these signals is fibroblast proliferation and collagen deposition.

It is inevitable that the evolution of IPF involves the interplay of numerous mediator cascades. Indeed, a host of specific mediators have been implicated in the pathogenesis of IPF (5-10). One group of mediators which has received relatively little attention in this condition are the 5-lipoxygenase (5-LO) metabolites of arachidonic acid. These substances, which include leukotrienes (LTs) $\mathrm{B}_{4}\left(\mathrm{LTB}_{4}\right)$ and $\mathrm{C}_{4}\left(\mathrm{LTC}_{4}\right)$, possess a wide spectrum of biological actions of a generally pro-inflammatory nature. Accordingly, they are thought to play important roles in both normal host defense as well as in a variety of inflammatory disorders $(11,12)$. Pulmonary disorders in which overproduction of LTs is implicated include asthma $(13,14)$ and the adult respiratory distress syndrome $(15,16)$. These lipid mediators are synthesized from arachidonic acid in a multistep pathway initiated by the enzyme 5-LO. In view of the pathophysiologic significance of LTs, understanding the molecular and cellular events involved in 5-LO activation has been an investigative priority in recent years (17). It is now recognized, for example, that activation of the enzyme entails its $\mathrm{Ca}^{2+}$-dependent translocation from a soluble locale to the nuclear envelope $(18,19)$.

1. Abbreviations used in this paper: 5-LO, 5-lipoxygenase; AM, alveolar macrophage; BAL, bronchoalveolar lavage; EIA, enzyme immunoassay; FLAP, 5-lipoxygenase activating protein; IPF, idiopathic pulmonary fibrosis; LT, leukotriene. 


\begin{tabular}{|c|c|c|c|c|c|c|c|c|c|c|c|}
\hline \multirow[b]{2}{*}{ Subject } & \multicolumn{2}{|c|}{ Used for } & \multirow[b]{2}{*}{ Age } & \multirow[b]{2}{*}{ Gender } & \multicolumn{3}{|c|}{ Smoking status } & \multirow{2}{*}{$\begin{array}{c}\text { Duration of } \\
\text { symptoms }\end{array}$} & \multirow[b]{2}{*}{$\mathrm{TLC}^{\S}$} & \multirow[b]{2}{*}{$\mathrm{D}_{\mathrm{L}} \mathrm{CO}^{\|}$} & \multirow[b]{2}{*}{$\mathrm{P}\left(\mathrm{A}-\mathrm{a} \mathrm{O} \mathrm{O}_{2}\right)^{\mathrm{q}}$} \\
\hline & Tissue & $\mathrm{AM}$ & & & $\mathrm{N} / \mathrm{F} / \mathrm{C}^{\ddagger}$ & pack-yr & yr quit & & & & \\
\hline & & & $y r$ & $M / F^{*}$ & & & & $y r$ & $\%$ pred & $\%$ pred & $m m ~ H g$ \\
\hline $\mathrm{a}$ & $\mathrm{x}$ & & 56 & M & $\mathrm{F}$ & 60 & 0.7 & 2 & 84 & 34 & 46 \\
\hline $\mathrm{b}$ & $\mathrm{x}$ & & 69 & $\mathrm{M}$ & $\mathrm{F}$ & 48 & 1 & 2 & 75 & 32 & 60 \\
\hline $\mathrm{c}$ & $\mathrm{x}$ & & 28 & $\mathrm{~F}$ & $\mathrm{~F}$ & 2 & 0.9 & 1 & 46 & 33 & 58 \\
\hline $\mathrm{d}$ & $\mathrm{x}$ & & 74 & $\mathrm{~F}$ & $\mathrm{~F}$ & 7 & 44 & 1 & 88 & 60 & 47 \\
\hline e & $\mathrm{x}$ & & 37 & $\mathrm{M}$ & $\mathrm{N}$ & & & 1 & 77 & 52 & 33 \\
\hline $\mathrm{f}$ & $\mathrm{x}$ & & 65 & M & $\mathrm{F}$ & 51 & 3 & 3 & 70 & 51 & 48 \\
\hline $\mathrm{g}$ & $\mathrm{x}$ & $\mathrm{x}$ & 59 & $\mathrm{~F}$ & $\mathrm{~N}$ & & & 1 & 72 & 62 & 42 \\
\hline $\mathrm{h}$ & $\mathrm{x}$ & $\mathrm{x}$ & 63 & $\mathrm{~F}$ & $\mathrm{~N}$ & & & 1 & 80 & 27 & 54 \\
\hline $\mathrm{i}$ & $\mathrm{x}$ & $\mathrm{x}$ & 37 & $\mathrm{~F}$ & $\mathrm{~F}$ & 5 & 7 & 1 & 82 & 47 & 33 \\
\hline $\mathrm{j}$ & & $\mathrm{x}$ & 76 & M & $\mathrm{N}$ & & & 1.5 & 61 & 29 & 54 \\
\hline $\mathrm{k}$ & & $\mathrm{x}$ & 66 & $\mathrm{M}$ & $\mathrm{F}$ & 12 & 31 & 5 & 83 & 36 & 41 \\
\hline 1 & & $\mathrm{x}$ & 69 & $\mathrm{~F}$ & $\mathrm{~F}$ & 7 & 13 & 2 & 49 & 60 & 42 \\
\hline $\mathrm{m}$ & & $\mathrm{x}$ & 67 & $\mathrm{~F}$ & $\mathrm{~N}$ & & & 2 & 69 & 43 & 38 \\
\hline mean & & & 58.9 & & & & & 1.8 & 72 & 43.5 & 45.8 \\
\hline SEM & & & 4.2 & & & & & 0.3 & 3.6 & 3.5 & 2.4 \\
\hline
\end{tabular}

$*$ male/female; ${ }^{\ddagger}$ never/former/current; ${ }^{\S}$ total lung capacity ( $\%$ of predicted value); ${ }^{*}$ diffusing capacity of lung for carbon monoxide $(\%$ of predicted value); ${ }^{\mathrm{I}}$ alveolar-arterial oxygen gradient at rest breathing room air.

The present investigation addressed the hypothesis that IPF is characterized by constitutive activation of 5-LO in the lower respiratory tract. Our results document that LT levels in lung homogenates of IPF patients exceeded those in control patients. Furthermore, the AM is one likely source of this constitutive overproduction of LTs in the IPF lung, on the basis of both in situ demonstration of 5-LO translocation to the nuclear envelope in AMs of IPF lung, as well as LT analysis of conditioned medium from cultured AMs.

\section{Methods}

Study populations. The IPF study group consisted of 13 previously untreated patients from the University of Michigan Specialized Center of Research in Interstitial Lung Disease project. All patients had clinical, radiographic, and physiologic findings consistent with IPF; those with findings suggestive of alternative diagnoses such as collagen-vascular disorders, asbestosis, hypersensitivity pneumonitis, sarcoidosis, or exposure to pneumotoxic substances, were excluded. Histologic confirmation of IPF was made by open lung biopsy in all patients. Their demographic and clinical characteristics are summarized in Table I. In nine of these patients (subjects a-i), lung tissue obtained at open biopsy was used for LT determinations and immunohistochemical analysis. In seven of these patients (subjects $\mathrm{g}-\mathrm{m}$ ), AMs obtained by bronchoalveolar lavage (BAL) were studied.

Three different control groups were used. (a) Eight patients with bronchogenic carcinoma undergoing surgical resection were used as a source of nonfibrotic lung tissue for LT determinations and immunohistochemical analysis; these patients served as a comparison population for the IPF patients (a-i) on whom tissue data were obtained. The demographic characteristics and histologic diagnoses of these cancer control patients are presented in Table II. (b) Six normal volunteers underwent BAL in order to obtain AMs for comparison to the AMs from IPF patients $\mathrm{g}-\mathrm{m}$; these subjects denied current or prior respiratory symptoms, any history of smoking, and recent (3 mo) upper respiratory infection or medication use, and had normal physical examinations of the lungs. This group was comprised of 4 males and 2 females, and their age was $28.7 \pm 2.3$ (mean \pm SEM) years. (c) Finally, lung tissue from one deceased individual whose lung was harvested as a potential transplant donor was also available for immunohistochemical analysis only. This individual was a 19 -yr-old male with no history of respiratory disease or smoking who died from a motor vehicle accident. This experimental protocol was approved by the University of Michigan Medical Center Institutional Review Board for Approval of Research Involving Human Subjects.

Lung tissue and preparation of lung homogenates. For IPF patients, separate surgical biopsy specimens were obtained from each of three different bronchopulmonary segments, and one small $\left(12 \mathrm{~mm}^{3}\right)$ piece from each segmental specimen was combined for subsequent homogenization. For bronchogenic carcinoma patients, tissue was isolated from an area of parenchyma distant from the tumor and was con-

Table II. Demographic and Clinical Characteristics of Lung Cancer Control Subjects

\begin{tabular}{|c|c|c|c|c|c|c|}
\hline \multirow[b]{2}{*}{ Subject } & \multirow[b]{2}{*}{ Age } & \multirow[b]{2}{*}{ Gender } & \multicolumn{3}{|c|}{ Smoking status } & \multirow[b]{2}{*}{ Histologic type } \\
\hline & & & $\mathrm{N} / \mathrm{F} / \mathrm{C}^{\ddagger}$ & pack-yr & yr quit & \\
\hline & $y r$ & $M / F^{*}$ & & & & \\
\hline 1 & 74 & M & $\mathrm{C}$ & 38 & & squamous \\
\hline 2 & 68 & $\mathrm{~F}$ & $\mathrm{C}$ & 42 & & squamous \\
\hline 3 & 56 & M & $\mathrm{F}$ & 38 & 5 & bronchoalveolar \\
\hline 4 & 59 & $\mathrm{~F}$ & $\mathrm{~F}$ & 26 & 3 & large cell undifferentiated \\
\hline 5 & 77 & M & $\mathrm{F}$ & 58 & 2 & squamous \\
\hline 6 & 53 & $\mathrm{~F}$ & $\mathrm{C}$ & 37 & & squamous \\
\hline 7 & 68 & M & $\mathrm{F}$ & 44 & 1.5 & small cell undifferentiated \\
\hline 8 & 34 & M & $\mathrm{C}$ & 8 & & undifferentiated \\
\hline mean & 61.1 & & & & & \\
\hline SEM & 4.9 & & & & & \\
\hline
\end{tabular}

* male/female; ${ }^{\ddagger}$ never/former/current. 
firmed to contain no neoplasm and to exhibit no fibrosis and normal architecture by histologic examination. Specimens from IPF and control lung were placed in $3 \mathrm{ml}$ of homogenizing buffer (PBS, $2 \mathrm{mM}$ phenylmethylsulfonyl fluoride, and $1 \mu \mathrm{g} / \mathrm{ml}$ each of antipain, aprotinin, leupeptin, and pepstatin A) and homogenized for $90 \mathrm{~s}$ with a Tissue Tearor (model 985-370; Biospec Products, Racine, WI). Homogenates were then sonicated for $30 \mathrm{~s}$ using a model 250 sonifier (Branson Ultrasonics Corp., Danbury, CT) at power level 1, 100\% duty cycle, and centrifuged for $10 \mathrm{~min}$ at $500 \mathrm{~g}$. The resulting supernatants were frozen at $-70^{\circ} \mathrm{C}$ for subsequent LT determination. Total protein was calculated for each sample using a microtiter plate modification (Pierce Biochemical, Rockford, IL) of the Bradford method (20) using bovine serum albumin as standard.

Isolation and culture of AMs. For isolation of AMs, IPF patients and healthy normal volunteers underwent fiberoptic bronchoscopy and bronchoalveolar lavage as previously described (10) using standard techniques. Lavage fluid was subsequently centrifuged at $500 \mathrm{~g}$ for $10 \mathrm{~min}$ and the resulting cell pellet was resuspended in PBS and cells enumerated using a hemacytometer. The percentage of lavage cells which were AMs was determined by examination of Diff-Quikstained cytocentrifuge preparations. Cells were centrifuged again and then resuspended in Dulbecco's modified Eagle's medium (DME) at $0.5 \times 10^{5} \mathrm{AMs} / 0.1 \mathrm{ml}$, and $0.2 \mathrm{ml}\left(10^{5} \mathrm{AMs}\right)$ were placed into each well of a 96-well plate and incubated for $2 \mathrm{~h}$ at $37^{\circ} \mathrm{C}$ in a humidified atmosphere of $5 \% \mathrm{CO}_{2}$ in air. Nonadherent cells were removed by washing twice with PBS, and the remaining adherent cells were incubated in DME for an additional $16 \mathrm{~h}$. These adherent cells were $97 \%$ AMs based on morphologic criteria and staining with nonspecific esterase. Following the $16 \mathrm{~h}$ incubation, the conditioned medium was frozen at $-70^{\circ} \mathrm{C}$ for subsequent analysis of LTs.

Quantitation of $\mathrm{LTB}_{4}$ and $\mathrm{LTC}_{4}$ in lung homogenates and in $\mathrm{AM}$ supernatants. Before determination of LTs in lung homogenates, potentially cross-reacting material was removed by extracting lipids with $\mathrm{C}_{18}$ Sep-Pak cartridges as previously described (21). This procedure has been used previously as a preparative step in assaying LT levels in bronchoalveolar lavage fluid $(22,23)$. The resulting lipid extract was evaporated to dryness under $\mathrm{N}_{2}$ and resuspended in assay buffer in order to quantitate LTs. AM culture supernatants were assayed directly without prior Sep-Pak extraction. In both cases, $\mathrm{LTB}_{4}$ and $\mathrm{LTC}_{4}$ levels were determined with enzyme immunoassay kits obtained from Cayman Chemical (Ann Arbor, MI). For each sample, the average of duplicate determinations was calculated.

Immunohistochemical staining of lung tissue for 5-LO protein. Lung tissue specimens from IPF or bronchogenic carcinoma patients were fixed in $4 \%$ paraformaldehyde for at least $24 \mathrm{~h}$. The tissue was then embedded in paraffin and 3-4- $\mu$ m-thick sections were cut and mounted on Superfrost/PLUS slides (Fisher Scientific, Pittsburgh, PA). Paraffin was removed from fixed sections with Americlear (Baxter Diagnostics, Deerfield, IL) and tissue was then rehydrated by sequential immersion for $1 \mathrm{~min}$ in descending concentrations of ethanol $(100 \% \times 2,50 \% \times 2,0 \%$ [PBS, $\mathrm{pH} 7.35] \times 2$. Staining was performed using the Vectastain ABC Elite immunohistochemistry system (Vector, Burlingame, CA). To reduce nonspecific binding, rehydrated tissue was incubated with Power Block (Biogenics, San Ramon, CA) for 15 min according to the manufacturer's instructions and then with $25 \%$ normal goat serum for $30 \mathrm{~min}$. Slides were incubated at $4^{\circ} \mathrm{C}$ for $24 \mathrm{~h}$ with either rabbit anti-human 5-LO antiserum (generously provided by Dr. Jilly Evans, Merck Frosst Canada, Pointe Claire-Dorval, Quebec) or nonimmune rabbit serum at dilutions of 1:1000-1:5000 in 25\% normal goat serum in PBS. Slides were then washed $2 \times$ for $10 \mathrm{~min}$ in PBS containing $2.5 \% \mathrm{NaCl}$ and $0.17 \%$ Triton X-100 (wash buffer) and biotinylated goat anti-rabbit IgG (1:600 dilution) was applied for $30 \mathrm{~min}$. Sections were again flooded with wash buffer for $10 \mathrm{~min} \times 2$, after which they were incubated with avidin-conjugated horseradish peroxidase reagent for $45 \mathrm{~min}$. After removal of the horseradish peroxidase reagent, sections were washed twice for $10 \mathrm{~min}$ each in wash buffer and then incubated with TrueBlue peroxidase substrate (KPL Laboratories, Gaithersburg, MD). Incubation times (usually 5-10 min) were determined by monitoring changes in specific signal and background staining over time via light microscopy. Sections were counterstained with Contrast Red (KPL Laboratories). The percentage of AMs in a given section of IPF or lung cancer control lung which demonstrated nuclear membrane localization of 5-LO was determined by evaluating all positively stained cells in 20 high power $(1000 \times)$ fields. In the single transplant donor lung, 85 high power fields were assessed.

For confocal microscopic examination, immunostaining was performed as described above, with the following modifications: blocking was carried out by incubating with $50 \%$ normal goat serum for 30 min, the primary antiserum dilution was 1:500-1:1000, and detection was with the Vectastain $\mathrm{ABC}$ alkaline phosphatase system (Vector) using Vector Red as the chromophore/fluorophore. Samples were visualized on a Bio-Rad MRC-600 laser confocal microscope at an excitation wavelength of $550 \mathrm{~nm}$. 1- $\mu \mathrm{m}$-thick optical sections were collected and relative intensity of fluorescence was converted to a pseudocolor display.

Histologic assessment of IPF lung tissue. Sections from each of the three bronchopulmonary segments sampled at open lung biopsy of IPF patients and stained with hematoxylin/eosin were evaluated separately for inflammation as well as fibrosis using a semi-quantitative

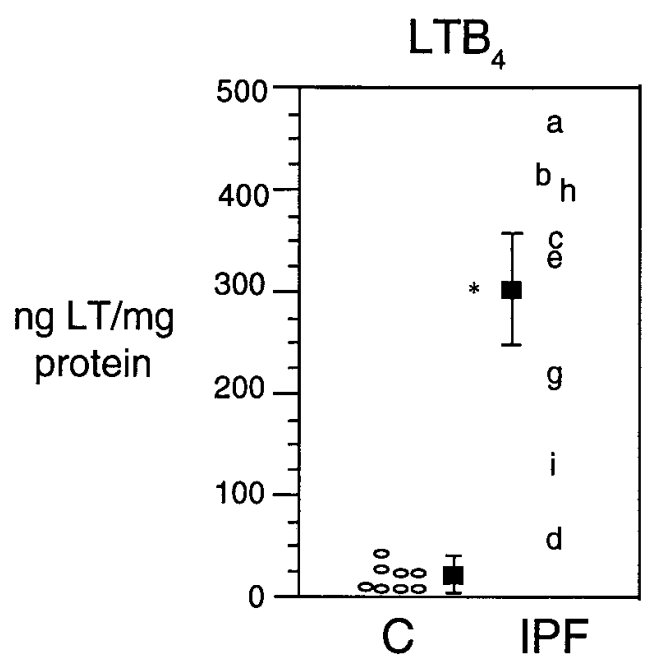

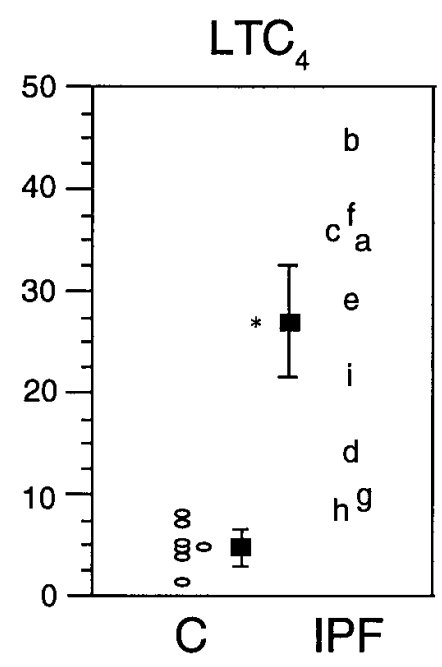

Figure 1. LT levels in homogenates from control and IPF lung. Homogenates were prepared from IPF lung (IPF) and from histologically nonfibrotic regions of control lung from patients with bronchogenic carcinoma $(C)$. Homogenates were extracted and assayed by EIA for $\mathrm{LTB}_{4}$ (left panel) and $\mathrm{LTC}_{4}$ (right panel). Results were normalized for the total protein concentration in each sample. Values from individual control patient samples are represented by open circles, and values from individual IPF patients by lowercase letters corresponding to their identifying designation in Table I. The mean \pm SEM for each group is represented by the closed square and bars. Note that one $\mathrm{LTB}_{4}$ value from the IPF group and one $\mathrm{LTC}_{4}$ value from the nonfibrotic control group are missing. $* P<$ 0.01 vs. C. 


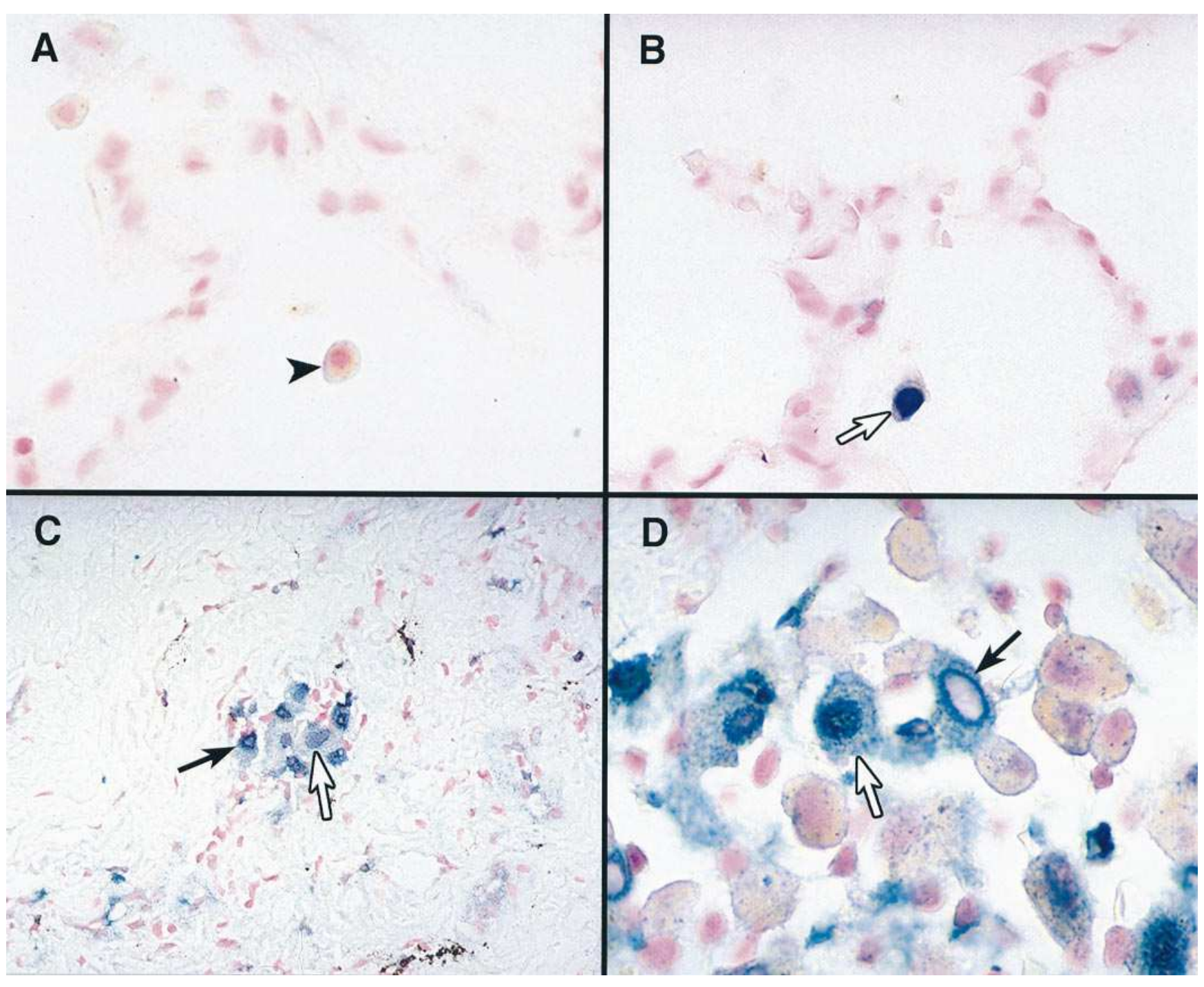

Figure 2. Immunohistochemical staining of 5-LO protein in control and IPF lung tissue. Sections from lung cancer control $(A$ and $B)$ and IPF $(C$ and $D$ ) lung tissue were incubated with either nonimmune rabbit serum $(A)$ or anti-5-LO antiserum $(B-D)$. Antigen was detected using an avidin-biotin-peroxidase system visualized with TrueBlue; Contrast Red was used as the counterstain. $A-C$ were photographed at low magnification and $D$ at high magnification. $A$ and $B$ are from the same specimen of control lung tissue; $C$ and $D$ are from two different IPF patients. The arrowhead in $A$ indicates an AM. In $B-D$, open arrows indicate representative cells demonstrating diffuse nuclear staining ("resting pattern") and filled arrows indicate representative cells demonstrating staining concentrated at the nuclear margin ("activated pattern").

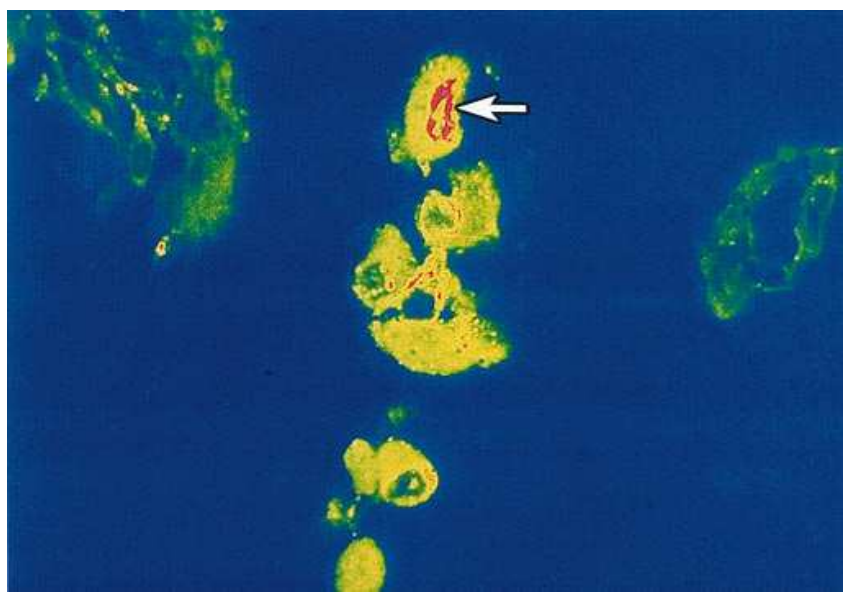

Figure 4. Pseudocolor display of 5-LO immunostaining in IPF lung tissue examined by confocal microscopy. A section of IPF lung tissue was incubated with anti-5-LO antiserum, and antigen was detected with Vector Red using confocal microscopy. Intensity of 5-LO fluorescence is displayed on a pseudocolor scale in which red represents most intense, and blue represents least intense staining. The arrow indicates one of a cluster of AMs exhibiting an "activated pattern" of 5-LO staining which is most intense at the nuclear margin. This can be compared to a very low-intensity staining alveolar wall lacking AMs in the upper left region of the photograph. 


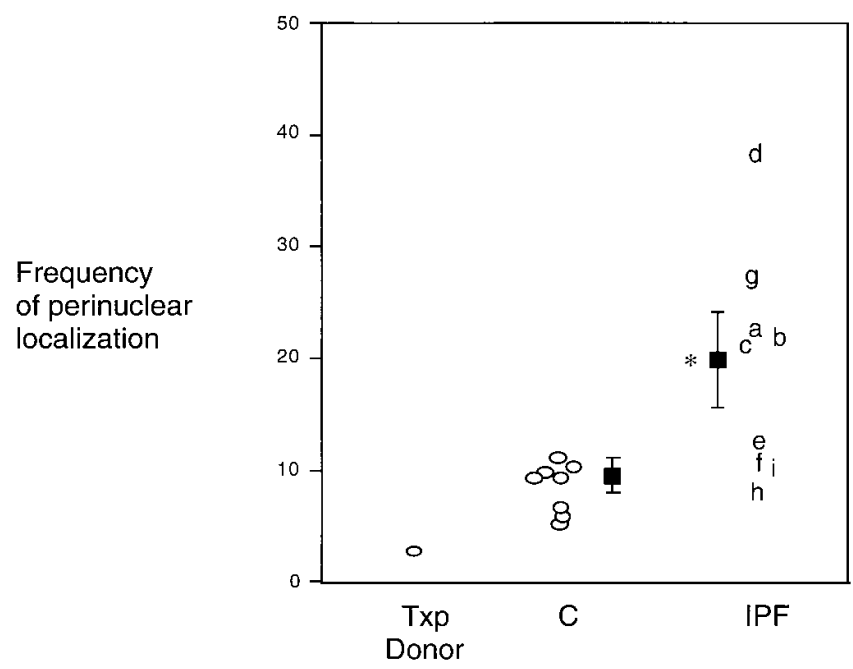

Figure 3. Frequency of perinuclear localization of 5-LO in AMs from control and IPF lung tissue. Sections of lung tissue from a single normal transplant donor lung ("Txp donor"), from cancer controls ("C"), and from IPF patients ("IPF") were subjected to immunohistochemical staining for 5-LO protein, and the frequency of a perinuclear ("activated") localization pattern among positively stained AMs was determined in 85,20 , and 20 high power $(1000 \times)$ fields, respectively. For controls, individual values are represented as open circles; for IPF patients, individual values are represented by lower case letters corresponding to their identifying designation in Table I. The mean ( \pm SEM for cancer controls and IPF) for each group is represented by the closed square (and bars). $* P<0.05$ vs. C.

scoring system previously described (24). The mean inflammation and fibrosis scores for each patient were then determined as the means of the individual values derived from each of the three segments. Although individual histologic scores are presented only for those patients whose tissue was analyzed for LTs (subjects a-i; depicted in Fig. 5), the mean inflammation (16.8 \pm 1.7$)$ and fibrosis (14.5 \pm 1.1$)$ scores for this subgroup were no different than those of the group from which AMs were harvested for culture (subjects g-m; inflammation, $15.1 \pm 1.1$; fibrosis, $14.0 \pm 1.0$ ).

Data analysis. LT levels in homogenates or in AM supernatants were expressed as the mean \pm SEM. Differences between the mean homogenate LT levels of IPF and cancer control groups were analyzed by the unpaired Student's $t$ test, and between mean AM supernatant levels by the Mann-Whitney rank sum test. Differences between IPF and cancer control lung tissue with respect to the mean frequency of nuclear membrane 5-LO staining were analyzed by the Mann-Whitney rank sum test. Correlations between homogenate LT levels and histologic indices were determined by linear regression analysis. A $P$ value $<0.05$ was taken as indicative of statistical significance.

\section{Results}

$\mathrm{LTB}_{4}$ and $\mathrm{LTC}_{4}$ levels in lung homogenates. The levels of $\mathrm{LTB}_{4}$ and $\mathrm{LTC}_{4}$ determined by immunoassay of lipid extracts of lung homogenates from patients with IPF and cancer controls are presented in Fig. 1. Both mediators were detectable in all patients, with $\mathrm{LTB}_{4}$ levels exceeding $\mathrm{LTC}_{4}$ levels in all samples. Notably, mean levels of both LTs were significantly $(P<0.01)$ higher in IPF lung homogenates than in control homogenates; the concentration of $\mathrm{LTB}_{4}$ was $\sim 15$-fold greater, and that of $\mathrm{LTC}_{4}, \sim 5$-fold greater than the control concentration. For both of these LTs, there was relatively little overlap between values in the IPF group and those in the control group. These data demonstrate that the 5-LO pathway of arachidonic acid metabolism is constitutively activated in lung tissue from patients with IPF.

Immunohistochemical staining of lung tissue for 5-LO protein. In a variety of cell types studied to date, in vitro stimulation of cellular LT synthesis has been associated with the translocation of 5-LO protein from its cytosolic or intranuclear locales in resting cells to the nuclear envelope $(18,19,25-27)$. We therefore reasoned that the constitutive activation of the 5-LO pathway in lung tissue from IPF patients might be associated with a similar accumulation of 5-LO protein at the nuclear envelope of the cell type or types responsible for LT overproduction in this disorder. To examine this possibility, paraffin-embedded sections of lung tissue from IPF patients, lung cancer controls, and a single transplant donor were subjected to immunohistochemical staining using nonimmune rabbit serum or rabbit anti-human 5-LO antiserum. Peripheral lung architecture was normal in sections from cancer control patients (Fig. 2, $A$ and $B$ ) and from the transplant donor (not shown). However, it was grossly abnormal in sections from IPF patients (Fig. 2, $C$ and $D$ ), demonstrating thickening of the alveolar septa, increased interstitial and intraalveolar cellularity, and increased numbers of fibroblasts as well as extracellular matrix deposition.

Incubation with nonimmune serum gave negligible staining in either control (Fig. $2 A$ ) or IPF lung (not shown). Anti-5-LO antiserum appeared to stain only AMs in sections of either cancer control (Fig. $2 \mathrm{~B}$ ), normal transplant donor (not shown), or IPF (Figs. 2, $C$ and $D$ ) lung tissue. The number of AMs staining positively for 5-LO was similar in the cancer control (15.9 \pm 5.2 cells/high power field) and IPF (11.9 \pm 2.3 cells/high power field) groups, but substantially greater than the number seen in the one normal transplant donor lung $(2.4$ cells/high power field). These results suggest that, although the uninvolved lung from cancer controls was architecturally normal, it did contain an increased number of AMs comparable to that observed in IPF.

Positively stained cells were then scored in order to determine the percentage exhibiting a pattern of nuclear membrane localization consistent with activation of 5-LO. The results from the various groups are presented in Fig. 3. In the truly normal transplant donor lung, most AMs showed diffuse intranuclear $>$ cytoplasmic staining, a pattern very similar to that which we have observed in human AMs isolated by bronchoalveolar lavage, mounted on slides, incubated with the same anti-5-LO antiserum, and examined by immunofluorescence microscopy $(19,27)$. An example of this pattern (in a section from a cancer control) is depicted in Fig. 2 B. A small number $(3.2 \%)$ of all stained AMs in the transplant donor lung exhibited a distinctly different staining pattern, in which the diffuse nuclear staining was diminished but intense staining could instead be visualized at the nuclear margin; this pattern was strikingly reminiscent of that seen in AMs activated in vitro $(19,27)$. In contrast to the transplant donor lung, however, an activated 5-LO staining pattern was identified in $19.2 \pm 3.3 \%$ (range, 8-38\%) of all positively stained AMs scored from the various IPF patients. Examples of perinuclear 5-LO staining from two different IPF patients are seen in Fig. 2, $C$ and $D$. Perinuclear staining of AM 5-LO was confirmed by confocal microscopy of Vector Red-stained sections of IPF lung (Fig. 4). Pseudocolor display revealed that the intensity of 5-LO 
A

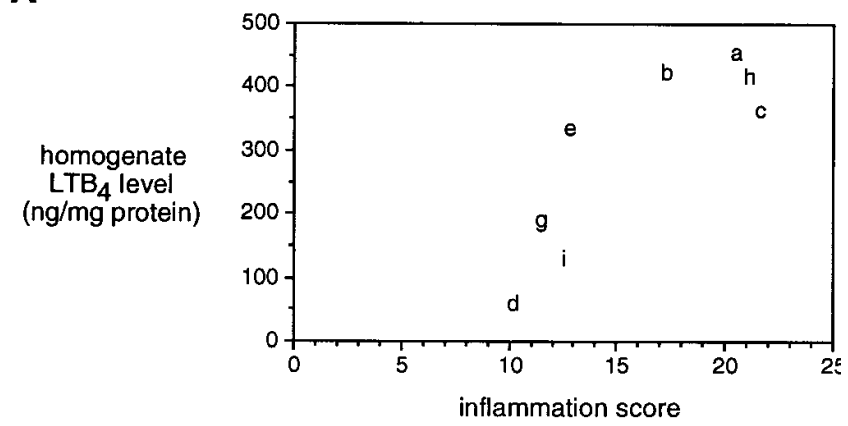

B

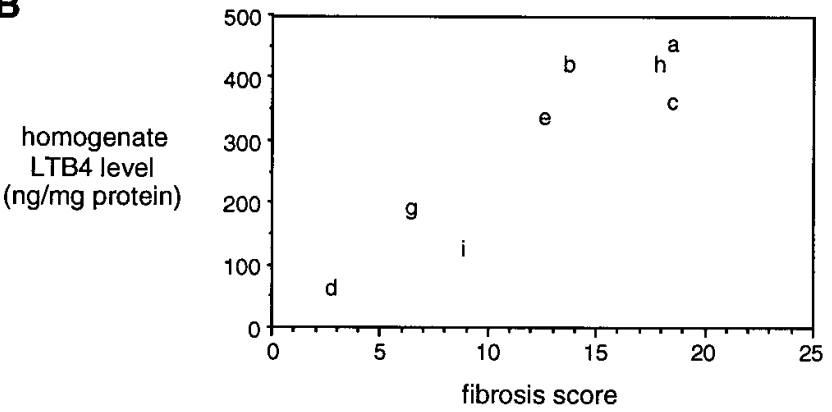

Figure 5. Correlations between homogenate $\mathrm{LTB}_{4}$ levels and histologic indices of inflammation and fibrosis in IPF lung tissue. Mean histologic scores for inflammation $(A)$ and fibrosis $(B)$, derived from three separate biopsy specimens, are plotted against homogenate $\mathrm{LTB}_{4}$ levels in lung tissue from each of 8 patients in the IPF study group. Individual values are represented by lower case letters corresponding to the identifying subject designations in Table I. Note that $\mathrm{LTB}_{4}$ data for one patient in this group is unavailable. Regression analysis indicated that the relationship between $\mathrm{LTB}_{4}$ and inflammation is described by the equation $y=27.271 \times-136.009 ; r=0.861$, $P<0.01$. The relationship between $\mathrm{LTB}_{4}$ and fibrosis is described by the equation $y=23.928 \times-2.867 ; r=0.926, P<0.001$.

staining was greatest at the nuclear margin of AMs, modest in the cytoplasm and in the nuclear interior of AMs, and minimal in a region of thickened alveolar wall lacking AM. These results are consistent with constitutive translocation of 5-LO to the nuclear envelope of many AMs in IPF lung, and suggest that the AM is one cell whose 5-LO enzyme is activated in this disorder. Fig. 3 also shows that lung tissue from cancer control patients likewise demonstrates a modest increase over normal transplant donor lung in the percentage of stained AMs with an activated localization pattern for 5-LO $(9.3 \pm 0.9 \%$; range, $6-12 \%)$. This is in keeping with the increased number of stained AMs counted in sections from these patients. Nonetheless, the percentage of cells exhibiting this activated pattern in cancer controls was significantly less $(P<0.05)$ than that seen in IPF patients, although some overlap was present between the two groups (Fig. 3). A comparison of the data presented in Figs. 1 and 3 indicates that the frequency of perinuclear staining of 5-LO in AMs and the LT content of the corresponding tissue did not appear to be closely related among individual IPF patients.

Correlations between homogenate $\mathrm{LTB}_{4}$ levels and histologic abnormalities. We sought to correlate levels of the predominant $\mathrm{LT}, \mathrm{LTB}_{4}$, with histologic indices of inflammation and fibrosis in the tissue obtained from IPF patients. Since $\mathrm{LTB}_{4}$ determinations were made on tissue specimens pooled from three different bronchopulmonary segments, mean histologic scores were similarly calculated for each patient from the 3 individual segmental values. Linear regression analysis was then performed between $\mathrm{LTB}_{4}$ level and both $(a)$ mean inflammation score and $(b)$ mean fibrosis score for the group of IPF patients. Homogenate $\mathrm{LTB}_{4}$ levels were significantly $(P<$ $0.01)$ correlated with mean inflammation scores $(r=0.861 ; \mathrm{y}=$ $27.271 \times-136.009$ ) (Fig. $5 A$ ). Interestingly, they were also highly correlated with mean fibrosis scores $(r=0.926 ; y=$ 23.928 $\times-2.867 ; P<0.001$ )(Fig. $5 B$ ). This is to be expected, since an inspection of the individual data points in Figs. 5, $A$ and $B$ indicates that inflammation and fibrosis scores paralleled each other in this group of patients.

Accumulation of $\mathrm{LTB}_{4}$ and $\mathrm{LTC}_{4}$ in AM conditioned medium. In view of the immunohistochemical evidence that IPF was characterized by an increased number of AMs exhibiting 5-LO activation, we isolated AMs from normal control subjects and IPF patients, purified them by adherence, and examined the accumulation of their major 5-LO metabolite, $\mathrm{LTB}_{4}$, during culture for $16 \mathrm{~h}$ in the absence of an exogenous stimulus. In some of these subjects (normals, $n=3$; IPF, $n=4$ ), this conditioned medium was also assayed for the minor AM metabolite, $\mathrm{LTC}_{4}$. $\mathrm{LTB}_{4}$ was detected in AM conditioned medium from only two of 6 normal control subjects. In contrast, it was detectable in medium from all seven IPF patients. Even when the undetectable $\mathrm{LTB}_{4}$ levels in normal volunteers were
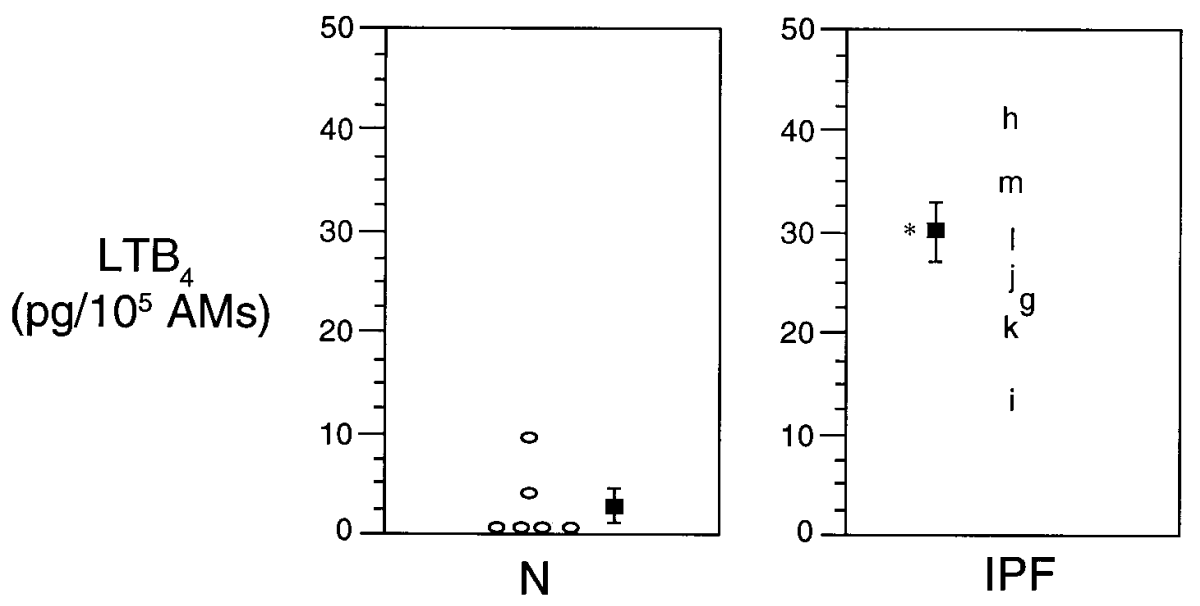

Figure 6. Constitutive $\mathrm{LTB}_{4}$ production by cultured AM from normal and IPF subjects. AM obtained by BAL from normal volunteers $(N)$ and IPF patients $(I P F)$ were purified by adherence and cultured for $16 \mathrm{~h}$ in the absence of a stimulus. Culture medium was collected and analyzed by EIA for $\mathrm{LTB}_{4}$, which was expressed as $\mathrm{pg} / 10^{5}$ plated AM. Individual values for normal controls are represented by open circles, and those for the IPF subjects by the lower case letters corresponding to the identifying designations in Table I. The mean \pm SEM for each group is represented by the closed square and bars. $* P<0.01$ vs. $\mathrm{N}$. 
considered to be equal to the lower limit of detection of the assay for the purpose of comparison of the group means, their AMs elaborated significantly less $(P<0.01) \mathrm{LTB}_{4}$ than did cells from IPF patients (Fig. 6). Similar findings were observed for $\mathrm{LTC}_{4}$ : this eicosanoid was undetectable in the conditioned medium from all 3 normal subjects in which it was assayed, but was detectable in all $4 \mathrm{IPF}$ patients examined $\left(11.4 \pm 2.9 \mathrm{pg} / 10^{5}\right.$ cells), albeit at a lower concentration than $\mathrm{LTB}_{4}$. These data confirm that the AM represents one cell type which contributes to the overproduction of both $\mathrm{LTB}_{4}$ and $\mathrm{LTC}_{4}$ observed in lung homogenates from IPF patients (Fig. 1).

\section{Discussion}

In the present study, we have demonstrated for the first time that levels of LTs $B_{4}$ and $C_{4}$ were greatly elevated in lung tissue obtained from open biopsies in patients with newly diagnosed IPF, as compared with levels measured in regions of nonfibrotic lung tissue obtained from patients undergoing resectional surgery for bronchogenic carcinoma. As these increases in LT levels were detected in tissue processed immediately after surgical removal and in the absence of exogenously added stimuli which might promote LT synthesis, these results suggest that the 5-LO metabolic pathway was constitutively activated in vivo to overproduce LTs. In view of the biological actions of LTs, discussed below, and the strong correlations identified between tissue LT levels and histologic degrees of inflammation and fibrosis, we speculate that these mediators may be important participants in the pathogenesis of IPF.

Two findings from our study point to the AM as one likely source of LT overproduction in the IPF lung. First, immunohistochemical staining of lung sections for 5-LO protein revealed that many AMs in IPF lung exhibited their most intense specific staining around the margins of the cell nuclei, consistent with translocation of enzyme to the nuclear envelope. This finding was noted with both conventional light microscopic as well as confocal microscopic examination. A nuclear envelope staining pattern, which is known to accompany 5-LO activation with resultant LT synthesis when cells are stimulated in vitro $(18,19,25-27)$, was rarely observed in AMs within normal lung harvested from a potential transplant donor, nor in other cell types in the IPF lung; however, it was observed to an intermediate degree in nonfibrotic lung from control patients with lung cancer. Second, AMs obtained from IPF patients by bronchoalveolar lavage and purified by adherence elaborated greater quantities of both $\mathrm{LTB}_{4}$ and $\mathrm{LTC}_{4}$ during overnight culture than did control AMs obtained from normal volunteer subjects. Of note, they did so in the absence of an exogenous stimulus, again suggesting that the 5-LO pathway of these cells had been activated in vivo. Interestingly, the ratio of $\mathrm{LTB}_{4}: \mathrm{LTC}_{4}(\sim 12: 1)$ found in IPF lung homogenates was similar to that produced by normal human AMs in response to exogenous stimuli $(28,29)$.

The basal overproduction of $\mathrm{LTB}_{4}$ and $\mathrm{LTC}_{4}$ by AMs from IPF patients is indicative of constitutive activation of the 5-LO enzyme; however, it is additionally possible that the steadystate expression per AM of 5-LO and/or 5-LO activating protein ("FLAP") (30) is upregulated in this disorder. Addressing this question will require further studies capable of detecting quantitative differences which cannot be accomplished by immunohistochemical analysis. Nonetheless, the fact that AM numbers are greatly increased in IPF (1) would certainly be expected to amplify the contribution of these cells; this may well be reflected in the measured levels of LTs in IPF lung homogenates. The in vivo stimulus for 5-LO activation in AM remains to be determined. However, one substance which is both sufficient to stimulate LT synthesis in AMs in vitro (31, 32) and is present in the circulation of some patients with IPF (33) is immune complexes. Alternatively, it is possible that cytokines such as interleukin-8, also reported to be increased in the lower respiratory tract of patients with $\operatorname{IPF}(9,10)$, could stimulate or prime AMs for enhanced LT synthesis (34).

It is interesting to note that elevated $\mathrm{LTB}_{4}$ levels have previously been reported in bronchoalveolar lavage fluid from patients with $\operatorname{IPF}(22,23)$ as well as asbestosis (35). Ozaki and colleagues (23), however, failed to detect an increase in $\mathrm{LTB}_{4}$ elaboration by AMs from IPF patients as compared with normal volunteers. This difference from our findings may reflect the fact that they harvested AM conditioned medium after only $4 \mathrm{~h}$, while we utilized a $16 \mathrm{~h}$ culture time. In view of the evidence that AMs overproduce LTs in IPF (present data) and asbestosis (35), it is not surprising that this abnormality would be reflected in analyses of lavage fluid. We chose to perform LT analyses on peripheral lung tissue homogenates instead, reasoning that this method too should detect LTs on the alveolar surface, albeit with less potential for dilution than lavage. Analysis of homogenates offers the added advantage of being able to detect those molecules found in the lung interstitium. Since $\mathrm{LTB}_{4}$ instilled into the airways of rats is rapidly transferred out of the alveolar compartment (36), LTs present in the interstitium might originate in either alveolar or interstitial compartments; on the basis of our findings, macrophages in either or both of these compartments can be postulated as the source of these eicosanoid mediators. Regardless of the cellular source, LT accumulation in the interstitium could be highly relevant in fibrotic disorders such as IPF, since it is primarily in this compartment that the characteristic exuberant fibroblast proliferation and collagen production take place.

The activation of cellular LT synthesis in vitro by incubation with experimental agonists has been associated with a redistribution of 5-LO protein from a soluble site to a membrane site $(37,38)$. The locale of soluble 5 -LO in resting cells has been shown to vary depending on the cell type, but both cytosolic and intranuclear pools have been recognized (19, 25, 26, $39)$. By contrast, loss of 5-LO from these soluble sites and an increase in 5-LO exclusively at the nuclear envelope has been a universal finding in a number of cell types, including neutrophils $(18,19,27)$ and AMs $(19,27)$, following agonist stimulation. This translocation event is thought to represent an essential early step in the activation of 5-LO. As the nuclear envelope has been shown to be an important site for both the liberation of arachidonic acid (40) and the localization of FLAP $(18,19,25)$, it is likely that translocation serves to bring 5-LO in close proximity to both its substrate and this important "helper" protein to allow concerted catalysis. The immunohistochemical demonstration of 5-LO localization at the nuclear envelope in AMs present within the lung of patients with IPF represents the first in situ evidence of enzyme translocation in any disease process. This finding serves to validate the veracity and relevance of information regarding 5-LO translocation heretofore derived exclusively from stimulation of isolated cells in vitro. It also suggests the possibility that in situ demonstration of translocation can be used as evidence of 5-LO activation in other disease processes where LTs are 
thought to be involved, such as asthma. Moreover, this method should have the capability to provide information regarding the cell type(s) in which 5-LO is activated in vivo.

Although the frequency of perinuclear localization of 5-LO ( $\sim 20 \%$ ) was much greater in AM in IPF lung than in the normal lung from a transplant donor $(\sim 3 \%)$, several limitations of these data must be acknowledged. First, we had access to only a single transplant donor lung for immunohistochemical analysis. Second, the frequency of an activated pattern of 5-LO localization in AM was also increased in sections of nonfibrotic lung resected at surgery for bronchogenic cancer, albeit significantly less than in IPF lung. Nonfibrotic lung from the cancer control patients also exhibited an increased total number of AMs per high power field as compared to the normal transplant donor lung. Thus, although this tissue was architecturally normal and nonfibrotic, it exhibited evidence of AM recruitment and activation as compared to truly normal lung tissue. Since half of these patients had ceased smoking for at least one year prior to resectional surgery, current smoking per se is unlikely to account for the increased number and state of activation of AMs in their lungs. Whether this instead reflects the consequences of prior smoking, of some degree of obstructive lung disease, or of lung cancer itself (41) remains to be determined. In any case, it is clear that this tissue from cancer patients serves as an imperfect control. Nonetheless, IPF lung significantly exceeded lung cancer control lung with respect to the content of LTs and the frequency of perinuclear localization of 5-LO; it is likely, however, that these data underestimate the differences in the degree of activation of 5-LO in IPF lung as compared to truly normal lung. Third, the frequency of an activated AM pattern of 5-LO localization varied substantially among IPF patients, with some exhibiting a 3- to 4-fold increase, and others a 7- to 12-fold increase over that seen in the normal transplant donor lung. Interpreting the possible significance, relationship to tissue LT content, and clinical and histologic correlates of such biological variation will require a far greater understanding of the process of 5-LO translocation and its consequences and reversibility than is presently available.

Although LTs have not classically been considered in fibrogenesis, their actions could be relevant to the pathogenesis of IPF in several ways. First, resident AM have a substantially greater capacity to synthesize $\mathrm{LTB}_{4}$ than do macrophages from other anatomic sites $(28,42,43)$. In addition, this molecule is a potent chemoattractant and functional activator of granulocytic cells, including neutrophils and eosinophils (44), and has been shown to account for an appreciable amount of the neutrophil chemotactic activity elaborated by AM (45); $\mathrm{LTB}_{4}$ synthesized by AMs could therefore play an important direct role in the leukocyte recruitment which characterizes IPF. Second, 5-LO metabolites enhance or play a permissive role in the elaboration of a variety of cytokines, including AM-derived fibroblast growth factor (46), tumor necrosis factor (47), interleukin-8 (48), interleukin-6 (49), and interferon- $\gamma$ (50); via this mechanism, they could amplify other pro-inflammatory and fibrogenic mechanisms. Third, LTs can exert direct effects on fibroblasts or other mesenchymal cells themselves. For example, they stimulate fibroblast chemotaxis (51), proliferation (52), and collagen synthesis (53). They have also been shown to augment the mitogenic effect of insulin-like growth factor on smooth muscle cells (54). The growth-promoting actions of LTs on lung fibroblasts are enhanced when endogenous fibroblast production of prostaglandin $\mathrm{E}_{2}$, which itself suppresses cell proliferation $(55,56)$, is pharmacologically inhibited $(52)$; in this regard, we have recently reported (57) that fibroblasts isolated from the lungs of patients with IPF have a diminished capacity to synthesize prostaglandin $\mathrm{E}_{2}$. This defect would therefore tend to magnify the effects on fibroblast proliferation of increased LT levels in the milieu of the IPF lung.

Correlations between histologic abnormalities and LT measurements of any kind have not been made previously in IPF. Given the potent neutrophil chemotactic effects of $\mathrm{LTB}_{4}$ in the lung (58), it is not surprising that lung homogenate $\mathrm{LTB}_{4}$ levels would be highly correlated $(r=0.861)$ with histologic evidence of inflammation. On the contrary, it is somewhat surprising that $\mathrm{LTB}_{4}$ levels were so highly correlated with histologic fibrosis scores $(r=0.921)$. It is generally assumed that fibrosis in IPF and other fibrotic disorders of the lung and elsewhere is the end-result of chronic inflammation. This paradigm might predict that by the time extensive histologic fibrosis is present, production of inflammatory mediators would have subsided. Although the number of subjects studied is admittedly small, our data suggest that LT overproduction is certainly ongoing in at least some patients with a severe degree of fibrosis.

Finally, the data presented here have implications for treatment of IPF. Standard treatment of IPF with corticosteroids is quite disappointing (59). Yet it is interesting to note the impressive efficacy demonstrated a decade ago for first-generation lipoxygenase inhibitors in attenuating the development of pulmonary fibrosis in a murine model of pulmonary fibrosis (60). In view of the recent development of far more potent and specific 5-LO inhibitors and LT receptor antagonists which have shown promise in the treatment of asthma in humans (61, 62), implicating LTs in the pathogenesis of IPF would provide the rationale for applying such specific targeted pharmacotherapy to this disorder as well. That LT overproduction appears to be a characteristic of even those with severe degrees of fibrosis means that the tendency of patients to reach clinical attention at fairly late stages in disease evolution would not exclude them from consideration of this therapeutic approach.

\section{Acknowledgments}

The authors thank Drs. Mark Orringer and Richard Whyte for providing lung tissue specimens, Janet Hampton for technical assistance, Kathy McClinchey for tissue sectioning, Dr. Thomas Brock for assistance with confocal microscopy, and Peggy Engel for secretarial support.

This work was supported by grants from the National Institutes of Health (RO1-HL47391 and Specialized Center of Research P50HL46487). Jerome Wilborn was the recipient of a Minority Investigator Research Supplement Award from the National Heart, Lung, and Blood Institute, Michael Coffey of a Clinical Investigator Development Award from the National Heart, Lung, and Blood Institute, Marc Bailie of an American Lung Association Research Fellowship, and Marc Peters-Golden of a Career Investigator Award from the American Lung Association.

\section{References}

1. Panos, R., and T. King. 1991. Idiopathic pulmonary fibrosis. In Immunologically Mediated Pulmonary Diseases. J.P. Lynch III, R.A. DeRemee, eds. J.B. Lippincott, Philadelphia, PA. 1-39.

2. Jones, R. 1991. The diagnosis of asbestosis. Am. Rev. Respir. Dis. 144: $477-478$.

3. King, T., Jr. 1993. Connective tissue disease. In Interstitial Lung Diseases. M.I. Schwarz, T.E. King, eds. Mosby-Year Book, St. Louis, MO. 271-288. 
4. Crystal, R., V. Ferrans, and F. Basset. 1991. Biologic basis of pulmonary fibrosis. In The Lung: Scientific Foundations. R.G. Crystal, J.B. West, editors. Raven Press, New York, NY. 2031-2046.

5. Martinet, Y., W. Rom, G. Grotendorst, G. Martin, and R. Crystal. 1987. Exaggerated spontaneous release of platelet-derived growth factor by alveolar macrophages from patients with idiopathic pulmonary fibrosis. N. Engl. J. Med. 317:202-209.

6. Khalil, N., R. O'Connor, H. Unruh, P. Warren, A. Kemp, O. Bereznay, K. Flanders, and A. Greenberg. 1990. Increased production and immunohistochemical localization of transforming growth factor beta (TGF- $\beta$ ) in idiopathic pulmonary fibrosis. Am. J. Respir. Cell Mol. Biol. 5:155-162.

7. Rennard, S., G. Hunninghake, P. Bitterman, and R. Crystal. 1981. Production of fibronectin by the human alveolar macrophage: mechanism for the recruitment of fibroblasts to sites of tissue injury in interstitial lung diseases. Proc. Natl. Acad. Sci. USA. 78:7147-7151.

8. Cantin, A., S. North, G. Fells, R. Hubbard, and R. Crystal. 1987. Oxidant mediated epithelial cell injury in idiopathic pulmonary fibrosis. J. Clin. Invest. 79:1665-1675.

9. Carre, P., R. Mortenson, T. King, Jr., P. Noble, C. Sable, and D. Riches. 1991. Increased expression of interleukin- 8 gene by alveolar macrophages in idiopathic pulmonary fibrosis: a potential mechanism for the recruitment and activation of neutrophils in lung fibrosis. J. Clin. Invest. 88:1802-1810.

10. Lynch, J.P. III., T. Standiford, M. Rolfe, S. Kunkel, and R. Strieter. 1992. Neutrophil alveolitis in idiopathic pulmonary fibrosis. Am. Rev. Respir. Dis. 145:1433-1439.

11. Lewis, R.A., K.F. Austen, and R.J. Soberman. 1990. Leukotrienes and other products of the 5-lipoxygenase pathway: biochemistry and relation to pathobiology in human disease. N. Engl. J. Med. 323:645-655.

12. Henderson, W., Jr. 1987. Eicosanoids and lung inflammation. Am. Rev. Respir. Dis. 135:1176-1185.

13. Drazen, J., J. O'Brien, D. Sparrow, S. Weiss, M. Martins, and E. Israel. 1992. Recovery of leukotriene $\mathrm{E}_{4}$ from the urine of patients with airway obstruction. Am. Rev. Respir. Dis. 146:104-108.

14. Taylor, G., P. Black, N. Turner, I. Taylor, N. Maltby, R. Fuller, and C. Dollery. 1989. Urinary leukotriene $\mathrm{E}_{4}$ after antigen challenge and in acute asthma and allergic rhinitis. Lancet. 1:584-588.

15. Stephenson, A., A. Lonigro, T. Hyers, R. Webster, and A. Fowler. 1988. Increased concentrations of leukotrienes in bronchoalveolar lavage fluid of patients with ARDS or at risk of ARDS. Am. Rev. Respir. Dis. 138:714-719.

16. Bernard, G., V. Korley, B. Swindell, A. Ford-Hutchinson, and P. Tagari. 1991. Persistent generation of peptido leukotrienes in patients with the adult respiratory distress syndrome. Am. Rev. Respir. Dis. 144:263-267.

17. Ford-Hutchinson, A., M. Gresser, and R. Young. 1994. 5-Lipoxygenase. Annu. Rev. Biochem. 63:383-417.

18. Woods, J., J. Evans, D. Ethier, S. Scott, P. Vickers, L. Hearn, S. Charleson, J. Heibein, and I. Singer. 1993. 5-Lipoxygenase and 5-lipoxygenase activating protein are localized in the nuclear envelope of activated human leukocytes. J. Exp. Med. 178:1935-1946.

19. Woods, J., M. Coffey, T. Brock, I. Singer, and M. Peters-Golden. 1995. 5-Lipoxygenase is located in the euchromatin of the nucleus in resting human alveolar macrophages and translocates to the nuclear envelope upon cell activation. J. Clin. Invest. 95:2035-2040.

20. Bradford, M. 1976. A rapid and sensitive method for the quantitation of microgram quantities of protein utilizing the principle of protein-dye binding. Anal. Biochem. 72:248-254.

21. Peters-Golden, M., and C. J. Shelley. 1988. Inhibitory effect of exogenous arachidonic acid on alveolar macrophage 5-lipoxygenase metabolism role of ATP depletion. J. Immunol. 140:1958-1966.

22. Wardlaw, A., H. Hay, O. Cromwell, J. Collins, and A. Kay. 1989. Leukotrienes, $\mathrm{LTC}_{4}$ and $\mathrm{LTB}_{4}$, in bronchoalveolar lavage in bronchial asthma and other respiratory diseases. J. Allergy Clin. Immunol. 84:19-26.

23. Ozaki, O., H. Hayashi, K. Tani, F. Ogushi, U. Yasuoka, and T. Ogura. 1992. Neutrophil chemotactic factor in the respiratory tract of patients with chronic airway diseases or idiopathic pulmonary fibrosis. Am. Rev. Respir. Dis. 145:85-91.

24. Cherniack, R., T. Colby, A. Flint, W. Thurlbeck, J. Waldron, L. Ackerson, and T. King, Jr. 1991. Quantitative assessment of lung pathology in idiopathic pulmonary fibrosis. Am. Rev. Respir. Dis. 144:882-900.

25. Peters-Golden, M., and R. McNish. 1993. Redistribution of 5-lipoxygenase and cytosolic phospholipase $\mathrm{A}_{2}$ to the nuclear fraction upon macrophage activation. Biochem. Biophys. Res. Commun. 196:147-153.

26. Chen, X.-S., T. Naumann, U. Kurre, N. Jenkins, N. Copeland, and C. Funk. 1995. cDNA cloning, expression, mutagenesis, intracellular localization, and gene chromosomal assignment of mouse 5-lipoxygenase. J. Biol. Chem. 270:17993-17999.

27. Brock, T.G., R.W. McNish, and M. Peters-Golden. 1995. Translocation and leukotriene synthetic capacity of nuclear 5-lipoxygenase in rat basophilic leukemia cells and alveolar macrophages. J. Biol. Chem. 270:21652-21658.

28. Balter, M., G. Toews, and M. Peters-Golden. 1989. Different patterns of arachidonate metabolism in autologous human blood monocytes and alveolar macrophages. J. Immunol. 142:602-608.

29. Balter, M., G. Toews, and M. Peters-Golden. 1989. Multiple defects in arachidonate metabolism in alveolar macrophages from young asymptomatic smokers. J. Lab. Clin. Med. 114:662-673

30. Miller, D.K., J.W. Gillard, P.J. Vickers, S. Sadowski, C. Leveille, J.A Mancini, P. Charleson, R.A.F. Dixon, A.W. Ford-Hutchinson, R. Fortin, J.Y. Gauthier, J. Rodkey, R. Rosen, C. Rouzer, I.S. Sigal, C.D. Strader, and J.F. Evans. 1990. Identification and isolation of a membrane protein necessary for leukotriene production. Nature (Lond.). 343:278-281.

31. Rankin, J., M. Hitchcock, W. Merrill, M. Bach, J. Brashler, and P. Askenase. 1982. IgE-dependent release of leukotriene $\mathrm{C}_{4}$ from alveolar macrophages. Nature (Lond.). 297:329-331.

32. Robbins, R., W. Russ, K. Thomas, J. Rasmussen, and H. Kay. 1987. Complement component C5 is required for release of alveolar macrophagederived neutrophil chemotactic activity. Am. Rev. Respir. Dis. 135:659-664.

33. Dreisin, R., M. Schwarz, A. Theofilopoulos, and R. Stanford. 1978. Circulating immune complexes in the idiopathic interstitial pneumonias. N. Engl. J. Med. 298:353-357.

34. Schroder, J.-M. 1989. The monocyte-derived neutrophil activating peptide (NAP/interleukin-8) stimulates human neutrophil arachidonate-5-lipoxygenase, but not the release of cellular arachidonate. J. Exp. Med. 170:847-863.

35. Garcia, J., D. Griffith, A. Cohen, and K. Callahan. 1989. Alveolar macrophages from patients with asbestos exposure release increased levels of leukotriene B. Am. Rev. Respir. Dis. 139:1494-1501.

36. Westcott, J.Y., T.J. McDonnell, and N.F. Voelkel. 1989. Alveolar transfer and metabolism of eicosanoids in the rat. Am. Rev. Respir. Dis. 139:80-87.

37. Rouzer, C.A., and S. Kargman. 1988. Translocation of 5-lipoxygenase to the membrane in human leukocytes challenged with ionophore A23187. J. Biol. Chem. 263:10980-10988.

38. Wong, A., S.M. Hwang, M.N. Cook, G.K. Hogaboom, and S.T. Crooke. 1988. Interactions of 5-lipoxygenase with membranes: Studies on the association of soluble enzyme with membranes and alterations in enzyme activity. Biochemistry. 27:6763-6769.

39. Brock, T.G., R. Paine, and M. Peters-Golden. 1994. Localization of 5-lipoxygenase to the nucleus of unstimulated rat basophilic leukemia cells. J. Biol. Chem. 269:22059-22066.

40. Capriotti, A., E. Furth, M. Arrasmith, and M. Laposata. 1988. Arachidonate released upon agonist stimulation preferentially originates from arachidonate most recently incorporated into nuclear membrane phospholipids. $J$. Biol. Chem. 263:10029-10034.

41. Vincent, J., M. Vermeer, W. Kort, and F. Zijlstra. 1990. The formation of thromboxane $\mathrm{B}_{2}$, leukotriene $\mathrm{B}_{4}$ and 12-hydroxyeicosatetraenoic acid by alveolar macrophages after activation during tumor growth in the rat. Biochim. Biophys. Acta. 1042:255-258.

42. Bigby, T.D., and M.J. Holtzman. 1987. Enhanced 5-lipoxygenase activity in lung macrophages compared to monocytes from normal subjects. J. Immunol. 138:1546-1550.

43. Peters-Golden, M., R.W. McNish, R. Hyzy, C. Shelly, and G.B. Toews. 1990. Alterations in the pattern of arachidonate metabolism accompany rat macrophage differentiation in the lung. J. Immunol. 144:263-270.

44. Ford-Hutchinson, A., M. Bray, and M. Doig. 1980. Leukotriene B B $_{4}$ a potent chemokinetic and aggregating substance released from polymorphonuclear leukocytes. Nature (Lond.). 286:264-265.

45. Martin, T., G. Raugi, T. Merritt, and W. Henderson, Jr. 1987. Relative contribution of leukotriene $\mathrm{B}_{4}$ to the neutrophil chemotactic activity produced by the resident human alveolar macrophage. J. Clin. Invest. 80:1114-1124.

46. Phan, S., B. McGarry, K. Loeffler, and S. Kunkel. 1987. Regulation of macrophage-derived fibroblast growth factor release by arachidonate metabolites. J. Leukocyte Biol. 42:106-113.

47. Dubois, C., E. Bissonnette, and M. Rola-Pleszczynski. 1989. Asbestos fibers and silica particles stimulate rat alveolar macrophages to release tumor necrosis factor. Autoregulatory role of leukotriene $\mathrm{B}_{4}$. Am. Rev. Respir. Dis. 139: 1257-1264.

48. Strieter, R., K. Kasahara, R. Allen, T. Standiford, M. Rolfe, F. Becker, S. Chensue, and S. Kunkel. 1992. Cytokine-induced neutrophil-derived interleukin-8. Am. J. Pathol. 141:397-407.

49. Thivierge, M., and M. Rola-Pleszczynski. 1992. Platelet-activating factor enhances interleukin-6 production by alveolar macrophages. J. Allergy Clin. Immunol. 90:796-802.

50. Johnson, H., and B. Torres. 1984. Leukotrienes: positive signals for regulation of $\gamma$-interferon production. J. Immunol. 132:413-419.

51. Mensing, H., and B. Czarnetzki. 1984. Leukotriene $B_{4}$ induces in vitro fibroblast chemotaxis. J. Invest. Dermatol. 82:9-12.

52. Baud, L., J. Perez, M. Denis, and R. Ardaillou. 1987. Modulation of fibroblast proliferation by sulfidopeptide leukotrienes: effect of indomethacin. $J$. Immunol. 138:1190-1195.

53. Phan, S., B. McGarry, K. Loeffler, and S. Kunkel. 1988. Binding of leukotriene $\mathrm{C}_{4}$ to rat lung fibroblasts and stimulation of collagen synthesis in vitro. Biochemistry. 27:2846-2853.

54. Cohen, P., J. Noveral, A. Bhala, S. Nunn, D. Herrick, and M. Grunstein. 1995. Leukotriene $\mathrm{D}_{4}$ facilitates airway smooth muscle cell proliferation via modulation of the IGF axis. Am. J. Physiol.(Lung Cell Mol. Physiol. 13). 269: L151-L157.

55. Bitterman, P., M. Wewers, S. Rennard, S. Adelberg, and R. Crystal. 1986. Modulation of alveolar macrophage-driven fibroblast proliferation by alternative macrophage mediators. J. Clin. Invest. 77:700-708. 
56. Fine, A., and R. Goldstein. 1987. The effect of $\mathrm{PGE}_{2}$ on the activation of quiescent lung fibroblasts. Prostaglandins. 33:903-913.

57. Wilborn, J., L. Crofford, M. Burdick, S. Kunkel, R. Strieter, and M. Peters-Golden. 1995. Fibroblasts isolated from patients with idiopathic pulmonary fibrosis have a diminished capacity to synthesize prostaglandin $\mathrm{E}_{2}$ and to express cyclooxygenase-2. J. Clin. Invest. 95:1861-1868.

58. Martin, T., B. Pistorese, E. Chi, R. Goodman, and M. Matthay. 1989. Effect of leukotriene $\mathrm{B}_{4}$ in the human lung: recruitment of neutrophils into the alveolar spaces without a change in protein permeability. J. Clin. Invest. 1989: $1009-1019$.

59. Turner-Warwick, M., B. Burrows, and A. Johnson. 1980. Cryptogenic fibrosing alveolitis: response to corticosteroid treatment and its effect on survival. Thorax. 35:593-599.
60. Phan, S., and S. Kunkel. 1986. Inhibition of bleomycin-induced pulmonary fibrosis by nordihydroguiaretic acid. The role of alveolar macrophage activation and mediator production. Am. J. Pathol. 124:343-352.

61. Cloud, M., G. Enas, J. Kemp, T. Platts-Mills, L. Altman, R. Townley, D. Tinkelman, T.J. King, E. Middleton, A. Sheffer, E.J. McFadden, and D. Farlow. 1989. A specific $\mathrm{LTD}_{4} / \mathrm{LTE}_{4}$ receptor antagonist improves pulmonary function in patients with mild chronic asthma. Am. Rev. Respir. Dis. 140:1336-1339.

62. Israel, E., P. Rubin, J. Kemp, J. Grossman, W. Pierson, S. Siegel, D. Tinkelman, J. Murray, W. Busse, A. Segal, J. Fish, H. Kaiser, D. Ledford, S Wenzel, R. Rosenthal, J. Cohn, C. Lanni, H. Pearlman, P. Karahalios, and J. Drazen. 1993. The effect of inhibition of 5-lipoxygenase by zileuton in mild-to-moderate asthma. Ann. Int. Med. 119:1059-1066. 